\title{
Influence of acoustic vibrations on the kinetics of liquid-phase chemical reactions involving associates
}

\section{Introduction}

In this communication, we report on the effect of external lowfrequency periodic mechanical influence on the stability of liquidphase bimolecular reactions with account for the association of both reagents. ${ }^{1,2}$ It is shown that a change in the character of oscillations of intermediate concentrations happens upon an increase in both the applied frequency and amplitude up to some critical values. Above critical amplitude, the reaction mixture no more contains trimers and the reaction is terminated. Our calculations have shown a possibility of controlling reaction rate in conditions of low-frequency acoustic influence.

\section{Mathematical model}

The kinetics of bimolecular reactions is described by non-linear differential equations that admit the formation of dissipative structures of intermediate concentration. ${ }^{3,4}$ We assumed that in solution reagent A could form dimmer $\mathrm{A}_{2}$ and trimmer $\mathrm{A}_{3}$; while reagent $\mathrm{B}$, monomer $\mathrm{B}$ and dimmer $\mathrm{B}_{2}$. Reaction product $\mathrm{C}$ is formed in the reaction $\mathrm{B}_{2}+$ $\mathrm{A}_{3} \rightarrow \mathrm{C}+\mathrm{B}+2 \mathrm{~A}^{2}$

The system of kinetic equations for this reaction can be written in the form:

$$
d\left[\mathrm{~A}_{2}\right] / d t=k_{1}[\mathrm{~A}]^{2}-k_{-1}\left[\mathrm{~A}_{2}\right]-k_{2}[\mathrm{~A}]\left[\mathrm{A}_{2}\right]+k_{-2}\left[\mathrm{~A}_{3}\right]-k_{3}[\mathrm{~A}]\left[\mathrm{A}_{2}\right]\left[\mathrm{A}_{3}\right],-----(1)
$$

$d\left[\mathrm{~A}_{3}\right] / d t=k_{2}[\mathrm{~A}]\left[\mathrm{A}_{2}\right]+k_{3}[\mathrm{~A}]\left[\mathrm{A}_{2}\right]\left[\mathrm{A}_{3}\right]-k_{-2}\left[\mathrm{~A}_{3}\right]-k_{4} k_{5}[\mathrm{~B}]_{2}\left[\mathrm{~A}_{3}\right]\left\{k_{-4}+\right.$ $\left.\mathrm{k}_{5}\left[\mathrm{~A}_{3}\right]\right\}^{-1}$

The action of acoustic wave with frequency $\mathrm{v}$ can be described by taking into account the stretching $(\sigma)$ developed in the system according to the following expression for rate constants $k(v)_{-\mathrm{i}}$ responsible for decomposition of associates: ${ }^{1,5}$

$$
\left.k(v)-i=k_{-0 i} \exp \left[\left(-E_{-\mathrm{i}}+\gamma \sigma\right) / R T\right]\right](\mathrm{i}=1,2)
$$

Where $\gamma$ is some structure-sensitive coefficient and $\sigma$ changes as $\sigma=\mathrm{P}_{0} \sin (2 \pi \mathrm{vt})$. At the initial stage, the formation of dimmers is governed by the value of $k(v)_{-i}$. It is almost convenient to create in a reaction system the density fluctuation, uniform in space, at the expense of external periodic force, influencing system an acoustic wave. At the action of a acoustic wave with a frequency $\mathrm{v}$ the stretching $\sigma$ and compression created in system lead to change of kinetic constants $k(v)$-and destruction of associates. This constant has view:

$$
\left.k(v)_{-i}=\mathrm{k}_{-0 \mathrm{i}} \exp \left[\left(-\mathrm{E}_{-\mathrm{i}}+\gamma \sigma\right) / R T\right]\right],(\mathrm{i}=1,2)
$$

Where $\gamma$-is a structure-sensitive coefficient, $\sigma-$ is the strenuous which changes under the law $\sigma=\mathrm{P}_{0} \sin (2 \pi v t)$. At the initial stage, the formation of dimmers is governed by the value of $k(v)_{-i}$.

\section{Results and discussion}

The problem was solved by mathematical modeling for the case $[\mathrm{A}]=[\mathrm{B}]=$ const as described elsewhere. ${ }^{2}$ The result is exemplified in Figure 1. An increase in oscillation frequency was found to suppress the oscillations in the concentration of intermediate and finally to terminate

\author{
Volume 2 Issue 3 - 2018
}

Kulagina TP, Smirnov LP,Andrianova ZS

Institute of Problems of Chemical Physics RAS, Russia

Correspondence: Kulagina TP, Institute of Problems of Chemical Physics RAS, Semenov prospect, Chernogolovka, Moscow region, 142432, Russia, Email tan@icp.ac.ru

Received: May 15, 2018| Published: June 18, 2018

the reaction. The model parameters used in calculations ${ }^{2}$ that yielded the data shown in Figure 1 were as follows: $\mu=k_{l}[\mathrm{~A}]^{2}=35 \mathrm{~mol}(\mathrm{ls})^{-1}$, $\mathrm{k}_{-2}=1 \mathrm{~s}^{-1}, \mathrm{u}=k_{3}[\mathrm{~A}]=01^{2} \mathrm{~mol}^{-2} \mathrm{~s}^{-1}, \mathrm{~m}=k_{4}[\mathrm{~B}]^{2}=200 \mathrm{~mol}(1 \mathrm{~s})^{-1}, k_{-1}=0.005 \mathrm{~s}^{-1}$, $\mathrm{a}=\mathrm{k}_{2}[\mathrm{~A}]=0.5 \mathrm{~mol}(\mathrm{ls})^{-1}$, and $\mathrm{g}=\mathrm{k}_{-4} \mathrm{k}_{5}-1=1.6 \mathrm{~mol} \mathrm{l}^{-1}$. In all calculations, it was adopted that $\mathrm{x}(0)=1.8 \mathrm{~mol} \mathrm{~s}^{-1}$ and $\mathrm{y}(0)=0.23 \mathrm{mols}^{-1}$.

The results have shown that the frequency and amplitude of external influence can be used as a tool for controlling the kinetics of chemical reactions, as well as for strengthening or weakening the self-oscillation of intermediate concentration, up to termination of the reaction. In our opinion, the influence of reagents association on the kinetics of chemical reactions deserves further basic and applied research. Advances in this line of research can be expected to provide theoretical backgrounds for controlling chemical kinetics and hence the structure/properties of products.

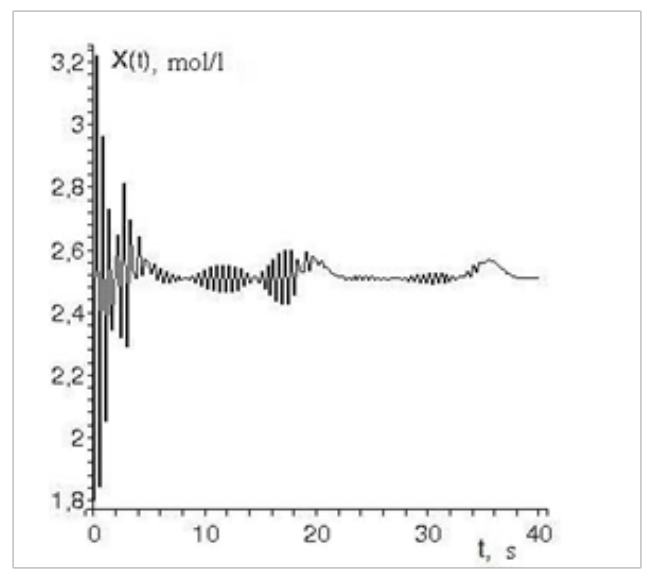

Figure I Concentration $\mathrm{x}=\left[\mathrm{A}_{2}\right]$ vs time $t$ in conditions of external acoustic influence (frequency $\omega=0.4 \mathrm{~s}^{-1}$, amplitude $\mathrm{P}=6$ ).

\section{Acknowledgements}

Work is performed on the theme of the state task of FASO № 00892014-0021.

\section{Conflict of interest}

Author declares that there is no conflict of interest. 


\section{References}

1. Smirnov LP, Kulagina TP. Features of the Kinetics of Chemical Reactions in a Nanostructured Liquid. Russian Journal of Physical Chemistry B. 2017;11(5):786-797.

2. Smirnov LP, Kulagina TP, Andrianova ZS. Influence of low frequency vibrations on the chemical reaction rate in the liquid phase for reagent association. Russian Chemical Bulletin. 2017;66(1): 2494-2496.
3. Ebeling W. Structure building bei irreversiblen Prozessen. Leipzig: Teubner; 1976.

4. Smirnov LP, Deyun EV, Baturin SM. Development of Microhetorogeneity Conditions in the Reaction System during Polyurethane Synthesis. Polymer Science Ser A. 1998;40(12):1238-1243.

5. Smirnov LP. Chemical Physics of Decomposition of Energetic Materials. Problems and Prospects. Russian Chemical Review. 2004;73(11):1121-1141. 